Canad. Math. Bull. Vol. 00 (0), 1977

\title{
EXTENSIONS OF ORDERABLE GROUPS
}

\author{
BY \\ R. BOTTO MURA AND A. H. RHEMTULLA
}

Introduction. The purpose of this note is to unify Theorem 4 of G. Baumslag [2] and a result of D. M. Smirnov in [6] in a more general setting. We prove the following result.

TheOREM. Let $A$ be a normal subgroup of a non-abelian free group $F, V a$ proper fully invariant subgroup of $A$ and $\underline{\underline{V}}$ the variety generated by $A / V$. If $A / V$ is orderable and $F / A$ has an infrainvariant system with factors in $\underline{\underline{V}}$ and right-orderable then $F / V$ is orderable.

Let $G=F / V, X=A / V$ and $Y=F / A$. Also let $R O$ denote the class of right-orderable groups. We fix this notation throughout the paper. Observe that if $X$ is a non-trivial orderable group, then $V \leq A^{\prime}$ and $\underline{\underline{V}}$ contains all abelian groups. Thus torsion-free abelian groups are in $R O \cap \underline{V}$ and the hypothesis of the theorem holds if $Y$ has an infrainvariant system with torsion-free abelian factors and $V=A^{\prime}$. This is Smirnov's result in [6]. If $Y$ is an ordered group, the convex subgroups of $Y$ form an infrainvariant system with torsion-free abelian factors. Thus the hypothesis of the theorem holds when $X$ and $Y$ are orderable. This is Theorem 4 in [2].

Definitions. We say that a group $H$ has an infrainvariant system with factors in a class $\underline{X}$ if $H$ has a set of subgroups $S=\left\{H_{\lambda} ; \lambda \in \Lambda\right\}$ such that (i) $\Lambda$ is a complete totally ordered set, (ii) $\langle e\rangle, G \in S$, (iii) if $\lambda<\mu$ then $H_{\lambda} \leq H_{\mu}$ and if $\mu$ is an immediate successor of $\lambda$ in the ordering of $\Lambda$ then $H_{\lambda} \quad H_{\mu}, H_{\mu} / H_{\lambda} \in X$, and (iv) for any $\lambda \in \Lambda$ and any $h \in H, H_{\lambda}^{h} \in S$. A group $H \in R O$ is the set $H$ can be ordered in such a way that for all $g, h, x$ in $H, g<h$ implies $g x<h x$. This is equivalent to saying that $H$ is isomorphic to a subgroup of the group of order preserving permutations of an ordered set (see [3]). If $H$ is a group and $Z$ a subset of $H$ then $S_{H}(Z)$ denotes the semigroup generated by $\left\{z^{h} ; z \in Z, h \in H\right\}$. If $K$ is a normal subgroup of a group $H$ then we say that $K$ is $H$-orderable if the set $K$ can be ordered in such a way that for all $x, y, z$ in $K, h$ in $H, x<y$ implies $x z<y z$ and $x^{h}<y^{h}$. This is equivalent to saying that given any finite set $x_{1}, \ldots, x_{n}$ in $K \backslash\langle e\rangle, e \notin S_{H}\left(x_{1}^{\varepsilon_{1}}, \ldots, x_{n}^{\varepsilon_{n}}\right)$ for a suitable choice of signs $\varepsilon_{i}= \pm 1$. If $H$ is $H$-orderable then we simply say $H$ is orderable and denote the class of such groups by $\mathrm{O}$.

Received by the editors January 24, 1977.

Research partially supported by the National Research Council of Canada. 
Proofs. We will use the following result which is a consequence of Theorem 1 in [2].

Proposition 1. (G. Baumslag). If $W=X$ wr $Y$, the standard restricted wreath product of $X$ and $Y$, and $B$ the base group of $W$ then given any finite set of elements $x_{1}, \ldots, x_{n} \in X \backslash\langle e\rangle$, there exists a homomorphism $\phi$ of $G$ into $W$ such that $\phi x_{1}, \ldots, \phi x_{n} \in B \backslash\langle e\rangle$.

Lemma 1. If $Y=F / A \in R O$ and $X=A / V \in O$ then $A / V$ is $G$ orderable. If $V=A^{\prime}$ then the converse is also true, that is if $A / V$ is $F / V$-orderable then $F / A \in R O$.

Proof. Let $W=X$ wr $Y$ and $B$ the base group of $W$. Under the given hypothesis, $B$ is $W$-orderable, since given any order on $X$ and right-order on $Y$ the corresponding lexicographic order of $B$ is a $W$-order. Suppose that $X$ is not $G$-orderable. Then there exist elements $x_{1}, \ldots, x_{n} \in X \backslash\langle e\rangle$ such that $e \in$ $S_{G}\left(x_{1}^{\varepsilon_{1}}, \ldots, x_{n}^{\varepsilon_{n}^{n}}\right)$ for all choices of signs $\varepsilon_{i}= \pm 1$. By Proposition 1 there is a homomorphism $\psi$ of $G$ into $W$ such that $\psi x_{1}, \ldots, \psi x_{n} \in B \backslash\langle e\rangle$. But then $S_{W}\left(\left(\psi x_{1}\right)^{\varepsilon_{1}}, \ldots,\left(\psi x_{n}\right)^{\varepsilon_{n}}\right)$ contains $e$ for all choices of signs $\varepsilon_{1}= \pm 1$, contradicting the fact that $B$ is $W$-orderable.

Conversely, in the case $V=A^{\prime}$, let $A / A^{\prime}$ be $F / A^{\prime}$-orderable. Since $C_{F}\left(A / A^{\prime}\right)=A([1]$, Theorem 1$), F / A$ is a group of order-preserving permutations of the ordered set $A / A^{\prime}$ and consequently is an $R O$-group.

Proof of the theorem. By hypothesis there exists an infrainvariant system $\sum=\left\{F_{\lambda}, \lambda \in \Lambda\right\}$ of subgroups connecting $A$ to $F$ with factors in $R O \cap \underline{Y}$. Let $\sum_{1}=\left\{v\left(F_{\lambda}\right), \lambda \in \Lambda\right\}$ where $v\left(F_{\lambda}\right)$ is the verbal subgroup of $F_{\lambda}$ corresponding to the variety $\underline{\underline{V}}$. Then $\sum_{1}$ is an infrainvariant system connecting $V$ to $F$. In fact

(i) $v\left(F_{\lambda}^{\mathrm{g}}\right)=\left(v\left(F_{\lambda}\right)\right)^{\mathrm{g}}$,

(ii) For any $B \subseteq \Lambda$, if $\bigcup_{\lambda \in B} F_{\lambda}=F_{\gamma}$ then $\bigcup_{\lambda \in B} v\left(F_{\lambda}\right)=v\left(F_{\gamma}\right)$ and

(iii) If $\bigcap_{\lambda \in B} F_{\lambda}=F_{\gamma}$ then $\bigcap_{\lambda \in B} v\left(F_{\lambda}\right)=v\left(F_{\gamma}\right)$.

(i) and (ii) are obvious and (iii) follows from a result of Dunwoody in [4]. Note that $\sum_{1}$ does not contain repetitions for $v\left(F_{\lambda}\right)=v\left(F_{\mu}\right)$ implies $F_{\lambda}=F_{\mu}$. Let $v\left(F_{\alpha}\right)<v\left(F_{\alpha+1}\right)$ be a jump in $\sum_{1}$ and let $N_{\alpha}=N_{F}\left(v\left(F_{\alpha}\right)\right)=N_{F}\left(v\left(F_{\alpha+1}\right)\right)$. Then $F_{\alpha}<F_{\alpha+1}$ is a jump in $\sum$. Since $v\left(F_{\alpha}\right)$ is fully invariant in $F_{\alpha}, N_{F}\left(F_{\alpha}\right) \leq N_{\alpha}$, conversely if $g \in N_{\alpha}$ and $F_{\beta}=F_{\alpha}^{g}$ then $v\left(F_{\beta}\right)=v\left(F_{\alpha}\right)$ and $\alpha=\beta$. Thus $N_{F}\left(F_{\alpha}\right)=$ $N_{\alpha}$. Since $F / A$ has a system with factors in $R O$, passing through $F_{\alpha}, N_{\alpha} / F_{\alpha}$ also has such a system and is therefore in $R O$. By Lemma $1, F_{\alpha} / v\left(F_{\alpha}\right)$ is $N_{\alpha} / v\left(F_{\alpha}\right)$ orderable. Since $F_{\alpha+1} / F_{\alpha} \in \underline{\underline{V}}, F_{\alpha} \geq v\left(F_{\alpha+1}\right)$ and therefore $v\left(F_{\alpha+1}\right) / v\left(F_{\alpha}\right)$ is also $N_{\alpha} / v\left(F_{\alpha}\right)$-orderable. By a theorem of Kokorin in [5], the system $\sum_{1}$ assures the orderability of $F / V$.

In the same way Theorem 1 of Smirnov in [7] can be modified to the following. 
If $Y$ has an infrainvariant system with factors in $\underline{\underline{V}}$ then $G$ has an infrainvariant system whose factors are subgroups of free $\underline{\underline{V}}$-groups.

\section{REFERENCES}

1. M. Auslander and R. C. Lyndon, Commutator subgroups of free groups. Amer. J. Math. 77, 929-931 (1955).

2. G. Baumslag, Wreath products and extensions. Math. Z. 81, 286-299 (1963).

3. P. M. Cohn, Groups of order automorphisms of ordered sets. Mathematika 4, 41-50 (1957).

4. M. J. Dunwoody, On verbal subgroups of free groups. Arch. Math. (Basel) 16, 153-157 (1965).

5. A. I. Kokorin, $\Gamma$-fully orderable and relatively convex subgroups of orderable groups. (In Russian) Sibirsk. Mat. Z. 7, 713-717 (1966).

6. D. M. Smirnov, Right-ordered groups. Algebra i Logika 5:6, 41-59 (1966).

7. D. M. Smirnov, On generalized solvable groups and their group rings. Mat. Sb. 109 (N.S. 67), 366-383 (1965).

Dép. Didactioue, F.S.E. UNIVERSITÉ LAVAL.

QúbBec, (Qứ) G1K 7P4 